Tendências em Matemática Aplicada e Computacional, 3, No. 2 (2002), 161-170.

(C) Uma Publicação da Sociedade Brasileira de Matemática Aplicada e Computacional.

\title{
Estimativa de Isotermas de Adsorção Gás-Líquido Usando a Abordagem de Problema Inverso
}

\author{
J. LUGON JUNIOR ${ }^{1}$, A.J. SILVA NETO ${ }^{2}$, Instituto Politécnico, Universidade do \\ Estado do Rio de Janeiro, Cx.P. 97282, 28601-970 Nova Friburgo, RJ, Brasil. \\ C.C. SANTANA ${ }^{3}$, Faculdade de Engenharia Química, Universidade Estadual \\ de Campinas-UNICAMP, Cx.P. 6066, 13081-970 Campinas, SP, Brazil.
}

Resumo. Neste trabalho é utilizado o método de Levenberg-Marquardt para estimar os coeficientes das isotermas de adsorção em interfaces gás-líquido em colunas de bolhas e espuma. São apresentados os resultados obtidos com o uso de dados simulados da concentração da albumina do soro bovino (BSA).

\section{Introdução}

Diversas indústrias produzem um volume significativo de efluentes líquidos ricos em material biológico. Estas indústrias podem ser classificadas basicamente em três grupos: $(i)$ processamento de alimentos; (ii) fermentados e destilados; e (iii) indústrias farmacêuticas [1]. Estes produtos devem ser retirados das águas residuais e alguns podem ser aproveitados na preparação de ração animal. Porém, normalmente estes compostos estão diluídos ou dissolvidos em baixas concentrações, o que dificulta seu aproveitamento. Recentemente o mecanismo de adsorção de proteínas em interfaces gás-líquido tem sido objeto de pesquisa, tanto teórica quanto experimental, devido à potencial utilização do fracionamento em colunas de bolhas e espuma como uma alternativa economicamente viável para a recuperação de compostos com atividade superficial a partir de soluções diluídas [2].

O sistema de fracionamento em colunas de bolha e espuma funciona basicamente através da injeção de um gás na base de uma coluna contendo a solução. As bolhas de gás formadas no distribuidor sobem até o topo da coluna e durante este trajeto adsorvem o soluto, que está dissolvido no líquido. Na região de espuma, ou seção de enriquecimento, formada acima da coluna de bolhas, é feita então a extração do material de interesse [3].

Para o melhor entendimento dos processos envolvidos, bem como a passagem da escala de laboratório para a escala industrial, é necessária a determinação das isotermas de adsorção em interfaces gás-líquido empregadas nos modelos matemáticos e

\footnotetext{
${ }^{1}$ lugon@iprj.uerj.br

2ajsneto@iprj.uerj.br

${ }^{3}$ santana@feq.unicamp.br
} 
numéricos desenvolvidos para a análise do sistema. Neste trabalho é empregada a abordagem de solução de problema inverso com este objetivo.

A análise de problemas inversos possui inúmeras aplicações relevantes em engenharia e medicina, bem como em outros ramos da ciência. Como exemplos da aplicação de problemas inversos em transferência de calor, citamos a estimativa da intensidade de fontes térmicas com dependência funcional no espaço e ou no tempo, a partir da medida transiente de temperaturas [4-6], e a estimativa da condição inicial de problemas transientes em transferência de calor por condução [7].

Os problemas inversos têm a característica de serem mal-postos [8], e diversas técnicas de solução têm sido desenvolvidas e aplicadas [9-13]. Usando o método de Levenberg-Marquardt [14] e medidas simuladas para a albumina do soro bovino (BSA) são estimados os parâmetros da isoterma e apresentados os intervalos de confiança para os mesmos.

\section{O Problema Direto}

O problema direto relativo à adsorção de bio-moléculas em interfaces gás-líquido em colunas de bolhas consiste essencialmente no cálculo da depleção, ou seja da redução das concentrações do composto de interesse com o tempo, quando são conhecidas as propriedades físico-químicas e os parâmetros do processo.

\subsection{Formulação Matemática do Problema Direto}

Será apresentado um modelo para descrever tanto o processo de adsorção quanto a depleção de soluto para uma solução de proteína. A taxa de adsorção de proteínas é dada por

$$
\dot{n}=\frac{6}{d_{b}}\left(v_{g} A\right) \Gamma,
$$

onde $d_{b}$ é o diâmetro das bolhas, $A$ é a área da seção transversal da coluna, $v_{g}$ é a velocidade superficial (vazão de gás dividida pela área da seção transversal da coluna $A$ ), e $\Gamma$ é a concentração superficial em excesso.

O fluxo de soluto para a superfície das bolhas pode ser calculado como

$$
\{\text { Fluxo Intersticial de Soluto }\}=k_{l}\left(C_{b}-C_{s}\right),
$$

onde $k_{l}$ é o coeficiente de transferência de massa, $a$ é a área intersticial das bolhas, $C_{b}$ é a concentração de soluto no líquido (bulk) e $C_{s}$ é a concentração de soluto junto às bolhas.

Fazendo um simples balanço de massa no volume diferencial representado na Figura 1, obtém-se

$$
\int_{0}^{\Gamma_{\text {saida }}} \frac{d \Gamma}{\left[C_{b}(t)-C_{s}(z, t)\right]}=\int_{0}^{H} \frac{\left(k_{l} a\right) d_{b}}{6 v_{g}} d z .
$$

Considerando que a velocidade e o diâmetro das bolhas são constantes ao longo da coluna, e que esta possui seção reta constante, obtém-se, das equações (2.1) e (2.3), 


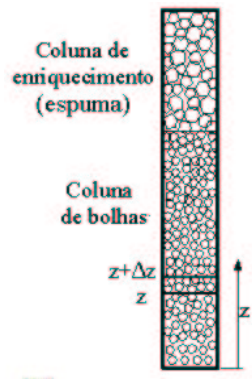

Figura 1: Coluna de fracionamento

$$
\frac{\partial \Gamma(z, t)}{\partial z}=\frac{\left(k_{l} a\right) d_{b}\left[C_{b}(t)-C_{s}(z, t)\right]}{6 v_{g}} .
$$

O coeficiente de transferência de massa pode ser calculado pela correlação de Özturk et al. [15]

$$
\left(\frac{\left(k_{l} a\right) d_{b}^{2}}{D_{i}}\right)=0,62\left(\frac{\mu_{f}}{\rho_{l} D_{i}}\right)^{0,5}\left(\frac{g \rho_{l} d_{b}^{2}}{\gamma}\right)^{0,33}\left(\frac{g \rho_{l} d_{b}^{3}}{\mu_{l}^{2}}\right)^{0,29}\left(\frac{v_{g}}{\sqrt{g d_{b}}}\right)^{0,68}\left(\frac{\rho_{g}}{\rho_{l}}\right)^{0,04},
$$

onde $D_{i}$ é o coeficiente de difusão do tensoativo, $\mu_{f}$ é a viscosidade do líquido, $\rho_{l}$ é a massa específica do líquido, $\gamma$ é a tensão superficial, $g$ é a aceleração da gravidade e $\rho_{g}$ é a massa específica do gás.

Гe $C_{s}$ estão relacionados por uma isoterma de adsorção, cuja formulação depende do composto de interesse [1]. Um modelo que permite caracterizar com muita precisão a depleção de proteínas como BSA (Albumina Bovina), Lysozima e $\beta$ Caseína,

$$
\Gamma_{t}=\Gamma_{1}+\Gamma_{2}=\frac{K_{1}(T) \exp \left(-\lambda \Gamma_{1}\right) C_{s}\left[1+K_{2}(T) \hat{a} C_{s}\right]}{\hat{a}\left[1+K_{1} \exp \left(-\lambda \Gamma_{1}\right) C_{s}\right]},
$$

onde $\Gamma_{1}$ e $\Gamma_{2}$ são as concentrações superfíciais em excesso da primeira e da segunda camada respectivamente, e $\hat{a}, \lambda, K_{1}$ e $K_{2}$ são coeficientes da isoterma.

Esta curva corresponde à modelagem termodinâmica desenvolvida por Guzman et al. [16], representando 2 camadas de adsorção. A primeira camada é equivalente à isoterma de Langmuir, podendo saturar, enquanto a segunda camada não apresenta saturação. Na Tabela 1 são apresentados os valores dos coeficientes $\hat{a}, K_{1}, K_{2}$ e $\lambda$ para três proteínas.

Aplicando novamente um balanço de massa, obtém-se [1]

$$
\frac{d C_{b}}{d t}=-k_{l} a\left(1-\varepsilon_{g}\right)\left(C_{b}-C_{s}\right),
$$

onde $\varepsilon_{g}$ é a fração volumétrica de gás, que pode ser calculada com a correlação adimensional de Kumar [17],

$$
\varepsilon_{g}=0,728 U-0,485 U^{2}+0,095 U^{3} \text {, onde } U=v_{g}\left[\frac{\rho_{l}^{2}}{\gamma\left(\rho_{l}-\rho_{g}\right) g}\right]^{\frac{1}{4}} .
$$


Tabela 1: Coeficientes das Isotermas para três proteínas

\begin{tabular}{|l|l|l|l|l|}
\hline Proteína & $\hat{a}\left[\mathrm{~m}^{2} / \mathrm{mg}\right]$ & $K_{1}[\mathrm{wt} \%]$ & $K_{2}\left[\mathrm{mg} /\left(\mathrm{m}^{2} \mathrm{wt} \%\right)\right]$ & $\lambda\left[\mathrm{m}^{2} / \mathrm{mg}\right]$ \\
\hline$\beta$-Caseína & 0,392 & 36.130 & 118,5 & $-0,674$ \\
\hline Lysozima & 0,338 & 86.330 & 607,4 & 0,641 \\
\hline BSA & 0,322 & 109.400 & 103,6 & 1,117 \\
\hline
\end{tabular}

\subsection{Solução do Problema Direto}

Combinando as equações (2.4) e (2.8) e usando uma condição inicial e uma condição de contorno do tipo, $\Gamma=0 \mathrm{em} z=0$ e $C_{b}=C_{b 0}$ em $t=0$, obtém-se a variação da concentração de soluto no líquido em função do tempo $C_{b}(t)$.

Santana e Carbonell [3] obtiveram uma solução analítica para o problema direto no caso de uma isoterma linear e os resultados obtidos apresentaram boa concordância com dados experimentais. Rosa [2] utilizou um procedimento numérico para obter uma solução adequada a isoterma de Langmuir e Lugon [18] adaptou este procedimento para atender às isotermas linear, de Langmuir e de 2 camadas. Primeiro modifica-se a equação (2.4) obtendo-se uma forma mais conveniente

$$
\int_{0}^{\Gamma_{s a i d a}} \frac{d \Gamma}{\left[C_{b}(t)-C_{s}(z, t)\right]}=\int_{0}^{H} \frac{\left(k_{l} a\right) d_{b}}{6 v_{g}} d z,
$$

onde $H$ é a altura da coluna de bolhas.

Fazendo um balanço global de massa na coluna de líquido levando em consideração as transferências que ocorrem na interface entre a coluna de bolhas e a coluna de espuma formada acima desta, obtém-se [2]

$$
\frac{d C_{b}}{d t}=f\left(t, C_{b}\right)=-\frac{6 v_{g}}{\left(1-\varepsilon_{g}\right) H d_{b}} \Gamma_{\text {saida }} .
$$

A equação (2.10) pode ser aproximada por diferenças finitas usando o método de Runge Kutta de segunda ordem, conhecido como método do ponto médio

$$
C_{b}^{n+1}=C_{b}^{n}+\Delta t f\left(t_{i}+\frac{\Delta t}{2}, C_{b}^{n}+\frac{\Delta t}{2} f\left(t_{i}, C_{b}^{n}\right)\right),
$$

com $C_{b}^{0}=C_{b}(t=0)=C_{b 0}$, onde $\Delta t$ é o passo no tempo, e $n$ indica o instante $t$ e $n+1$ o instante $t+\Delta t$.

\section{O Problema Inverso}

Silva Neto e Moura Neto [19] classificaram os problemas inversos levando em consideração a dimensão do modelo matemático e a dimensão dos objetos a serem estimados. Esta classificação está apresentada na Tabela 2.

O problema inverso aqui considerado consiste na estimativa dos coeficientes da isoterma de adsorção a partir de medidas experimentais das concentrações do soluto 
Tabela 2: Classificação dos problemas inversos

\begin{tabular}{|c|c|c|}
\hline \multirow{2}{*}{ Modelo Matemático } & \multicolumn{2}{|c|}{ Estimativa } \\
\cline { 2 - 3 } & Dimensão finita (constante) & Dimensão infinita (função) \\
\hline Dimensão finita $^{1}$ & Tipo I & Não se aplica \\
\hline Dimensão infinita $^{2}$ & Tipo II & Tipo III \\
\hline & ${ }^{1}$ por exemplo: sistema de equações algébricas \\
2 & por exemplo: equação diferencial parcial ou equação integro-diferencial
\end{tabular}

dissolvido no líquido em função do tempo. Tem-se, portanto um problema inverso do Tipo II, ou seja, o modelo matemático é de dimensão infinita e a estimativa de dimensão finita.

\subsection{Formulação do Problema Inverso}

Para o processo de transferência de massa através da adsorção de tensoativos na interface gás-líquido em colunas de bolhas, segundo a formulação apresentada na Seção 2.1, tem-se o interesse em estimar simultaneamente, ou não, os coeficientes da isoterma $\left(\hat{a}, \lambda, K_{1}\right.$ e $\left.K_{2}\right)$.

Como a quantidade de medidas experimentais é superior ao número de incógnitas a serem estimadas, o problema inverso é resolvido como um problema de otimização de dimensão finita onde busca-se minimizar o funcional de resíduos quadrados

$$
S=\sum_{i=1}^{N}\left(\frac{C_{b i}(\vec{P})-Y_{i}}{\sigma_{i}}\right)^{2}=\vec{F}^{T} W \vec{F},
$$

onde $Y_{i}$ é a concentração de soluto medida no líquido, $C_{b i}$ é a concentração calculada, $M$ é o número de medidas experimentais, $\vec{P}$ é o vetor de incógnitas, $\sigma_{i}$ é o desvio padrão dos erros das medidas de concentração, $W$ é a matriz de peso contendo o inverso das variâncias na diagonal e $F_{i}=C_{b i}-Y_{i}, i=1,2, \ldots, M$.

\subsection{Solução do Problema Inverso}

Para a solução do problema inverso de transferência de massa descrito na seção anterior foi usado o método de Levenberg-Marquardt [14]. Este método tem sido usado com sucesso na solução de problemas inversos do Tipo II [5,21].

Buscando minimizar a norma $S$ é primeiramente escrita a equação do ponto crítico

$$
\frac{d S}{d \vec{P}}=\frac{d}{d \vec{P}}\left(\vec{F}^{T} W \vec{F}\right)=0 \quad=>\quad J^{T} W \vec{F}=0,
$$

onde $J$ é a matriz Jacobiana, com os elementos $J_{p s}=\frac{\partial C_{b p}}{\partial P_{s}}$ sendo $p=1,2, \ldots M$ e $s=1,2, \ldots N$, e $N$ é o número total de incógnitas.

Aplica-se então uma expansão em série de Taylor a $\vec{F}(\vec{P})$, mantendo apenas os termos até a primeira ordem, 


$$
\vec{F}(\vec{P}+\Delta \vec{P})=\vec{F}(\vec{P})+J \Delta \vec{P} .
$$

Utilizando a expansão acima na Eq. (13) obtém-se

$$
J^{T} W J \Delta \vec{P}=-J^{T} W \vec{F}(\vec{P}) .
$$

No método de Levenberg-Marquardt é somado à diagonal da matriz $J^{T} J$ um valor $\beta$ para auxílio na convergência do método, sendo este valor reduzido durante o procedimento iterativo que é executado até que se satisfaça um critério de convergência estabelecido a priori.

Para a utilização no procedimento iterativo, a equação (3.4) é escrita de uma forma mais conveniente,

$$
J^{T} W J \Delta \vec{P}=-J^{T} W \vec{F}(\vec{P}) .
$$

O procedimento iterativo é iniciado com uma estimativa para os parâmetros, $\vec{P}^{0}$, sendo novas estimativas obtidas com $\vec{P}^{n}=\vec{P}^{n-1}+\Delta \vec{P}^{n}$, calculando-se as correções $\Delta \vec{P}^{n}$ com a equação (3.5), até que o critério de convergência

$$
\Delta \vec{P}^{n}=-\left[\left(J^{n}\right)^{T} W J^{n}+I \beta\right]^{-1}\left(J^{n}\right)^{T} W \vec{F}\left(\vec{P}^{n}\right)
$$

seja satisfeito, onde $\varepsilon$ é um número pequeno, e.g. $10^{-5}$, $I$ é a matriz identidade e $n$ é o contador de iterações

Os elementos da matriz Jacobiana, bem como o termo do lado direito da equação (3.6), são calculados a cada iteração usando a solução do problema direto empregando as estimativas para as incógnitas obtidas na iteração anterior.

Deve ser observado que o problema dado pela equação (3.6) é diferente do problema original dado pela equação (3.5). Ao longo do procedimento iterativo busca-se, portanto, reduzir o valor do fator de amortecimento, $\beta$, para que ao se atingir a convergência tenha-se a solução do problema original.

\subsection{Intervalos de Confiança}

Os intervalos de confiança para as estimativas $\vec{P}^{n}$ são calculados usando o procedimento desenvolvido por Gallant [20]. Empregando a notação utilizada por Huang e Özisik [21],

$$
\left|\frac{\Delta P_{n}^{k}}{P_{n}^{k}}\right|<\varepsilon, \quad n=1,2, \ldots, N,
$$

onde $\vec{C}_{b}$ é o vetor contendo os elementos $\left\{C_{b_{i}}\right\}$ e $\sigma$ é o desvio padrão dos erros experimentais.

Assumindo uma distribuição normal para os erros experimentais, e $99 \%$ de confiabilidade, os limites de intervalos de confiança para as estimativas $P_{s}, s=$ $1,2, \ldots, N$, são calculados por Flach e Özisik [22]

$$
\sigma_{\vec{P}}=\sigma\left\{\operatorname{diag}\left[\frac{\partial \vec{C}_{b}^{T}}{\partial \vec{P}} \frac{\partial \vec{C}_{b}}{\partial \vec{P}^{T}}\right]^{-1}\right\}^{1 / 2}
$$




\section{Projeto de Experimentos}

O uso de experimentos bem projetados deve resultar em estimativas mais precisas e confiáveis. Duas ferramentas disponíveis para este projeto envolvem a análise dos coeficientes de sensibilidade e do determinante da matriz $X^{T} X$.

\subsection{Coeficientes de Sensibilidade}

As derivadas parciais das variáveis de estado, em nosso caso a concentração de tensoativos, em relação aos parâmetros que se deseja estimar, por exemplo os parâmetros da isoterma ou o diâmetro das bolhas, são chamadas de coeficientes de sensibilidade. Através da análise destes coeficientes pode-se concluir se o propósito de estimar certos parâmetros tem chance de ser bem sucedido. De fato estes permitem o projeto de experimentos otimizados onde buscam-se medidas experimentais que sejam influenciadas significativamente pelas grandezas que se deseja determinar.

Dowding et al. [23], analisaram o cálculo e o uso de coeficientes de sensibilidade em problemas de condução de calor, demonstrando como estes fornecem informações fundamentais sobre os efeitos dos parâmetros nas respostas dos modelos.

Para a obtenção de boas estimativas, é fundamental que os coeficientes de sensibilidade tenham valores relativamente elevados, fazendo com que as respostas do modelo reflitam pequenas mudanças nas grandezas a serem estimadas.

Quando são estimadas várias grandezas diferentes simultaneamente, são utilizados os coeficientes escalonados ou modificados.

Quando estimam-se diversos parâmetros de um modelo, os efeitos de cada um deles nas concentrações devem ser diferentes, ou seja independentes. Se os coeficientes de sensibilidade de dois parâmetros têm a mesma forma, significa que eles afetam as concentrações da mesma maneira e então é difícil distinguir suas influências e portanto estimá-los.

Na Figura 2 são apresentados os Coeficientes de Sensibilidade para um experimento com BSA, concentração inicial $C_{b 0}=316 \mathrm{~g} / \mathrm{m}^{3}$, altura da coluna de líquido $H=0,60 \mathrm{~m}$, diâmetro das bolhas $d_{b}=1,0 \mathrm{~mm}$ e velocidade superficial $v_{g}=0,14 \mathrm{~cm} / \mathrm{s}$.

No caso representado na Figura 2, trata-se de um experimento não ótimo, observa-se a dificuldade de estimar os coeficientes $\lambda$ e $K_{1}$ devido a sua pequena sensibilidade e os coeficientes $\hat{a}$ e $K_{2}$ simultaneamente pela semelhança nas formas de suas sensibilidades.

\subsection{Determinante da Matriz $X^{T} X$}

Quando se pretende estimar dois ou mais parâmetros simultaneamente, existem diversos critérios de otimização possíveis, todos dados em termos de $X^{T} X$, podemos citar: (i) maximização do determinante de $X^{T} X$; (ii) maximização do menor autovalor de $X^{T} X$; (iii) maximização do traço de $X^{T} X[10]$. 


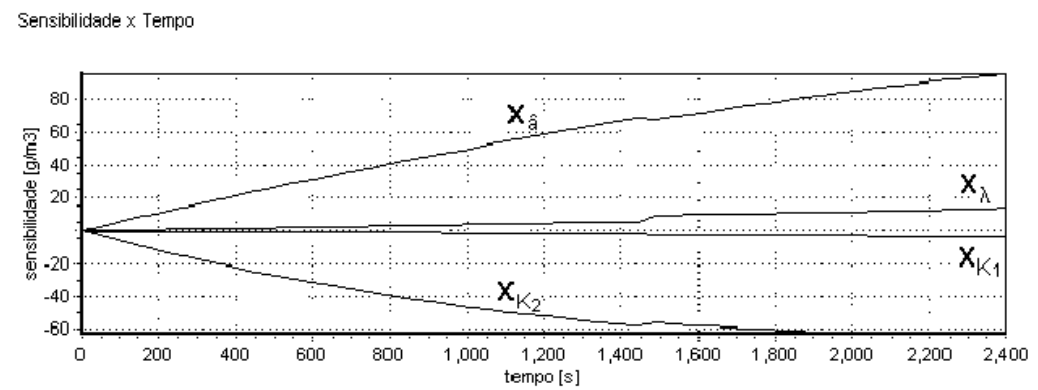

Figura 2: Coeficientes de Sensibilidade para BSA

Em nosso estudo foi utilizado o primeiro, ou seja, a maximização do determinante da matriz $X^{T} X$. Na Figura 3, estão representados os valores deste determinante, para estimativa simultânea de $\hat{a}$ e $K_{2}$, variando-se condições de operação dos experimentos.

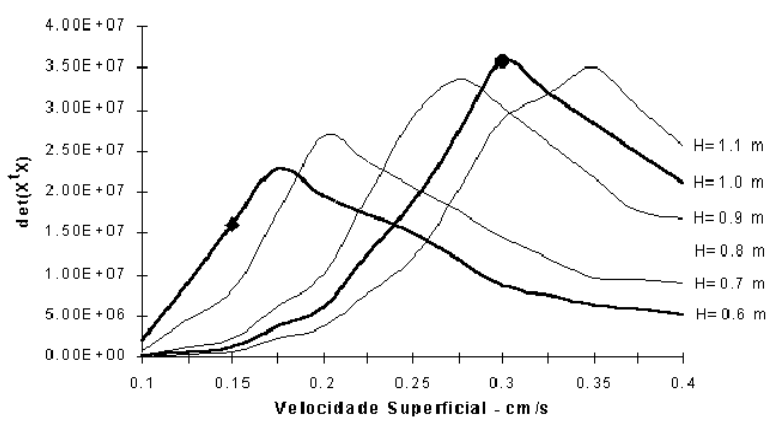

Figura 3: Determinante da matriz $X^{T} X$

\section{Resultados e Conclusões}

Com objetivo de comparar o resultado das estimativas com uso de um experimento ótimo e com um outro não otimizado, efetuou-se a estimativa de $\hat{a}$ e $K_{2}$ com uma série de experimentos gerados numericamente, através da adição de erros randômicos. Os resultados destas estimativas estão representados na Figura 4. Pode-se observar que os intervalos de confiança para ambos os parâmetros são menores para o experimento otimizado e as estimativas são mais próximas ao valor original.

Os resultados obtidos até o momento para as estimativas das isotermas de adsorção são extremamente satisfatórios. Foram também considerados outros tensoativos tais como brometo de hexadeciltrimetil amônio (CTAB) e cytochrome C. 

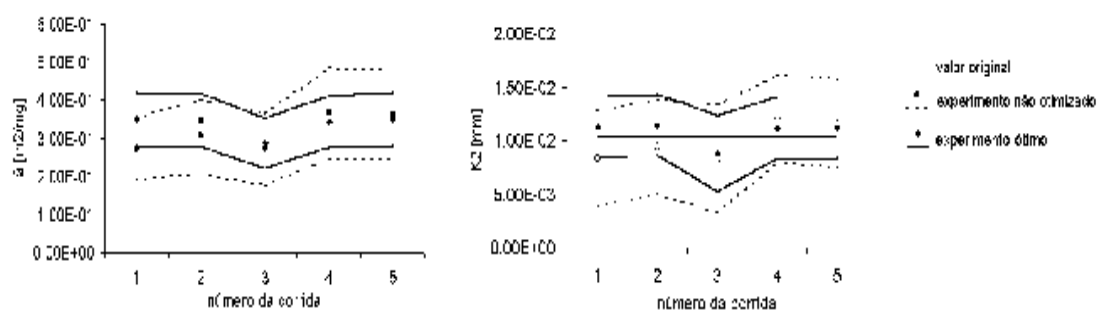

Figura 4: Resultados das estimativas de $\hat{a}$ e $K_{2}$ para diferentes corridas

\section{Referências}

[1] C.C. Santana, Adsorptive bubble separation process as a means of reducing surface-active contaminants in industrial wastewaters, 1994, Revista Brasileira de Engenharia - Química Especial, 5 (1994).

[2] P.T.V. Rosa, "Fracionamento de Proteínas e Outros Tensoativos em Colunas de Bolhas e de Espuma", Tese de Doutoramento, Faculdade de Engenharia Química, Universidade Estadual de Campinas, 1996.

[3] C.C. Santana e R.G. Carbonell, Adsorptive bubble separation as a means of reducing surface-active contaminants in industrial wastewaters, em "Proceedings of the Inter. Symp. on Heat and Mass Transfer", Mexico, pp. 1-11, 1993.

[4] J. Su e A.J. Silva Neto, Two-dimensional inverse heat conduction problem of source strength estimation in cylindrical rods, Applied Mathematical Modelling, 25 (2001), 861-872.

[5] A.J. Silva Neto e M.N. Özisik, The estimation of space and time dependent strength of a volumetric heat source in a one-dimensional plate, Int. J. Heat and Mass Transfer, 37, No. 6 (1994), 909-915.

[6] A.J. Silva Neto e J. Su, The estimation of heat source strength in plate-type fuel elements with cladding, em "Proc. $3^{\text {rd }}$ International Conference on Inverse Problems in Engineering: Theory and Practice", Port Ludlow, USA, 1999.

[7] A.J. Silva Neto e M.N. Özisik, An inverse heat conduction problem of unknown initial condition, em "Proc. 10th International Heat Transfer Conference", Vol. 6, pp. 421-426, Brighton, Inglaterra, 1994.

[8] A.N. Tikhonov e V.Y. Arsenin, "Solutions of Ill-Posed Problems", Winston, Washington D.C., 1977

[9] O.M. Alifanov, E.A. Artyukhin e S.V. Rumiantsev, "Extreme Methods for Solving Ill-Posed Problems with Applications to Inverse Heat Transfer Problems", Springer Verlag, 1995 
[10] J.V. Beck e K.J. Arnold, "Parameter Estimation in Engineering and Science", Wisley, New York, 1977.

[11] E. Hensel, "Inverse Theory and Applications for Engineers", Prentice Hall, Englewood Cliffs, New Jersey, 1991.

[12] D.A. Murio, "The Mollification Method and the Numerical Solution of Ill-Posed Problems", John Wiley \& Sons, New York, 1993.

[13] A.J. Silva Neto e N.C. Roberty, A abordagem fonte-detetor na solução de problemas inversos em meios participantes, em "Anais do V Congresso de Engenharia Mecânica Norte-Nordeste", Vol. III, pp. 340-347, 1998.

[14] D.W. Marquardt, 1963, An algorithm for least-squares estimation of nonlinear parameters, J. Soc. Industr. Appl. Math., 11 (1963), 431-441.

[15] S.S. Özturk, A. Schumpe e W.D. Deckwer, Organic liquids in a bubble column: holdups and mass transfer coefficients, AIChE Journal, 33 (1987), 1473-1480.

[16] R. Guzman, R.G. Carbonell e P.K. Kilpatrick, The adsorption of proteins to gas-liquid interfaces, Journal of Colloid and Interface Science, 114, No. 2 (1986), 536-547.

[17] A. Kumar, T.T. Dagaleesan, G.S. Ladha e H.E. Hoelscher, Bubble swarm characteristics in bubble columns, Canadian Journal of Chemical Engineering, 54 (1976), 503-510.

[18] J. Lugon Jr., "Um Problema Inverso de Transferência de Massa no Processo de Separação por Adsorção Gás-Líquido em Colunas de Bolhas e Espuma", Tese de Mestrado, Instituto Politécnico, Univ. do Estado do Rio de Janeiro, 2000.

[19] A.J. Silva Neto e F.D. Moura Neto, Escolha de Modelos-Problemas Inversos em Engenharia, Notas de Aula do Minicurso Técnico MC05 no "XXII Congresso Nacional de Matemática Aplicada e Computacional", Santos, São Paulo, 1999.

[20] A.R. Gallant, "Nonlinear Statistical Models", Wiley, New York, 1987.

[21] C.H. Huang, e M.N. Özisik, A direct integration approach for simultaneously estimating spatially varying thermal conductivity and heat capacity, Int. J. Heat and Fluid Flow, 11 (1990), 262-268.

[22] G.P. Flach e M.N. Özisik, Inverse heat conduction problem of simultaneously estimating spatially varying thermal conductivity and heat capacity per unit volume, Numer. Heat Transfer, 16 (1989), 249-266.

[23] K.J. Dowding, B.F. Blackwell e R.J. Cochan, Applications of sensitivity coefficients for heat conduction problems, Numerical Heat Transfer, Part B, 36 (1999), 33-55. 